\title{
¿Cómo medir el subsidio soviético a la economía cubana? Una nueva propuesta
}

\author{
por \\ Bruno Sovilla ${ }^{1}$ \\ Universidad Autónoma de Chiapas, México
}

\begin{abstract}
El comercio entre la ex Unión Soviética (URSS) y Cuba hasta el derrumbe del campo socialista, ha sido objeto de numerosos estudios que lo han tratado de medir en dólares estadounidenses, sin tomar en cuenta las peculiaridades de las relaciones entre los países socialistas miembros del CAME (Consejo de Ayuda Mutua Económica). En este trabajo, utilizando fuentes oficiales (de la oficina de estadística y del Banco Nacional de Cuba), tratamos de subsanar los errores metodológicos cometidos, proponiendo una nueva medición de la ayuda soviética a Cuba.
\end{abstract}

PALABRAS ClAVE: subsidio; tipo de cambio; azúcar; petróleo; reexportaciones.

Cómo Citar Este Artículo / Citation: Sovilla, Bruno, “Cómo medir el subsidio soviético a la economía cubana? Una nueva propuesta", Revista de Indias, LXXXI/283 (Madrid, 2021): 833-864. https://doi.org/10.3989/revindias.2021.024.

\section{INTRODUCCIÓN}

El primer tratado comercial entre Cuba y la Unión Soviética (URSS) estableció la compra por parte de los soviéticos de 425.000 toneladas de azúcar en los primeros meses de 1960 , de 575.000 en la segunda mitad del año, y de un millón anuales en los cuatro años siguientes. Se estableció un precio de

1 brunosov@hotmail.com, bruno.sovilla@unach.mx, ORCID iD: https://orcid.org/00000002-1148-6670 
poco más de 4 centavos por libra, que en ese periodo resultó superior al del mercado mundial ${ }^{2}$. A mitad de 1960, frente a la eliminación de la cuota azucarera $^{3}$ por parte del gobierno norteamericano, la URSS vino en ayuda y se empeñó en comprar una cantidad de azúcar superior a la que Cuba perdió en el mercado norteamericano ${ }^{4}$. En 1961 los precios pagados por la URSS resultaron preferenciales 5 y cuando el precio en el mercado mundial aumentó en 1963, los soviéticos también lo adecuaron hasta 6,11 centavos por libra, manteniéndolo hasta 1972. Cabe precisar que estos datos oficiales son en dólares, sin embargo solo el $20 \%$ de los pagos eran en moneda convertible, el resto era en rublos transferibles, aunque de hecho se trataba de trueques ${ }^{6}$.

En 1960 la URSS ofreció a Cuba el primer crédito al desarrollo, equivalente a 100 millones de dólares, a una tasa de interés del 2,5\% que había que devolver en 12 años ${ }^{7}$, empezando la que sería una fuente de financiamiento importante para la economía caribeña.

Un segundo tratado comercial estipulado en enero de $1964^{8}$, estableció la venta de 24,1 millones de toneladas de azúcar entre 1965-70, al precio definido anteriormente y que resultó preferencial (superior al del mercado mundial) de 6,11 centavos por libra ${ }^{9}$. Después de este acuerdo fue eliminada la obligación de pago de un $20 \%$ en moneda convertible, sin embargo, la idea de estabilizar el precio y la producción de azúcar resultó ventajosa para Cuba, al menos hasta 1971, dada la caída del precio del dulce en el mercado mundial (entre 1966-68 se llegó a pagar 1,8-1,9 centavos por libra). En realidad las entregas de azúcar por parte de Cuba a la URSS fueron poco más de la mitad de las que se habían pactado ${ }^{10}$, como consecuencia de los graves problemas que la economía cubana sufrió en la última parte de esa década.

2 Torres, 1981: 288.

3 Los Estados Unidos compraban cada año una cantidad previamente acordada de azúcar cubano, a precios preferenciales, conocida como «cuota azucarera». Con el triunfo de la revolución cubana (en enero 1959) y las primeras medidas que afectaron a las propiedades de empresas norteamericanas en la isla, incluyendo la nacionalización de las refinerías que se negaron a procesar el petróleo crudo importado de la Unión Soviética, las relaciones entre los dos gobiernos se hicieron más tensas, llegando a la eliminación de la cuota azucarera y culminando en la declaración de embargo hacia la isla por el presidente Eisenhower en octubre de 1960.

${ }^{4}$ Mesa Lago, 2000: 178.

5 Rodríguez, 1992b: 8.

${ }^{6}$ Rodríguez, 1992a: 61.

${ }^{7}$ Rodríguez, 1986: 30. Mesa Lago, 2000: 178.

8 Torres, 1981: 288. Mesa Lago, 2000: 202.

9 Castro, 1977: 226.

${ }^{10}$ Entre 1965 y 1970 Cuba entregó a la URSS solo 12,85 millones de toneladas de azúcar, de las 24,1 que habían sido acordadas. Eso por haber producido solo 35,1 millones de tone- 
La siguiente sesión de acuerdos tuvo lugar en Moscú, en diciembre de 1972 e incluyó el primer protocolo de intercambio comercial a mediano plazo (1973-75). Se fijó en 11 centavos la libra el precio pagado por los soviéticos para el azúcar, y en 5.000 dólares la tonelada el níquel, el segundo producto de exportación de Cuba a la URSS ${ }^{11}$. En aquella ocasión, Cuba recibió una primera prórroga para el pago de cualquier tipo de crédito recibido hasta ese momento, incluyendo un periodo de gracia para poderlo pagar, sin cálculo de intereses en este mismo periodo ${ }^{12}$. En 1984 hubo otra renegociación, que prorrogó aún más el periodo de pago, hasta $1991^{13}$, año en el cual la URSS dejó de existir.

En 1975 se abrió una fase superior ${ }^{14}$ en las relaciones comerciales entre los dos países, introduciendo un mecanismo de indexación del precio de los principales bienes comerciados, con el objetivo de mantener estables los términos de intercambio.

Sucesivamente, en 1984, se firmó un programa de colaboración económica y científico-técnica hasta el año $2000^{15}$ que tuvo solo una aplicación parcial por el derrumbe del campo socialista en 1989 y la desaparición de la misma URSS dos años después.

Antes de 1975 los precios de los productos intercambiados en el CAME se fijaban según la fórmula de Bucarest ${ }^{16}$, por un quinquenio. Terminado el periodo, se modificaban en base a la variación del precio en el mercado mundial. Sin embargo, esta fórmula cesó antes de su revisión oficial, en enero de 1975. Desde 1972 el precio del petróleo pagado por Cuba a la URSS empezó a aumentar, mientras se había mantenido constante en el quinquenio anterior, como consecuencia del incremento del precio en 1971. Después de otro importante aumento del precio del petróleo entre 1973-74, cuando se multiplicó por cuatro, el costo de oportunidad para los soviéticos de las exportaciones petroleras a Cuba creció mucho, sugiriendo un cambio en la fórmula para introducir una mayor flexibilidad. Desde 1975 la tasa de

ladas, de los 47 millones previstos en el plan (cálculos con datos de Mesa Lago, 1973: 558. Anuario Estadístico de Cuba, 1972: 214-15).

11 Rodríguez, 1992b: 3.

12 Ibidem: 6.

13 Banco Nacional de Cuba, 1985: 3.

14 Rodríguez, 1986: 14.

15 Rodríguez, 1992b: 7. Mesa Lago, 2000: 242.

16 Según el acuerdo de Bucarest (1958) los países socialistas miembros del Consejo de Ayuda Mutua Económica (CAME), se reunían cada cinco años para fijar los precios de las mercancías que intercambiaban entre ellos y estos precios se mantenían hasta la siguiente negociación. 
variación del precio del petróleo que Cuba importaba de la URSS, se determinaba como media móvil de la tasa de variación del precio del mercado mundial en los 5 años anteriores y cada año se modificaba (entrevista del autor a la investigadora Helena Álvarez del Instituto Nacional de Investiga-

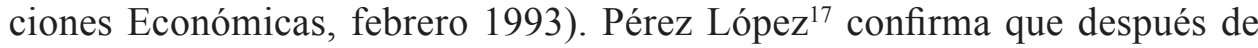
1975 se cambió la manera de fijar los precios: «parece que la URSS haya pasado a implementar ajustes anuales de los precios de las exportaciones de petróleo a los miembros del CAME basados en la media móvil sobre 5 años de los precios del mercado mundial, antes de 1974». Marrese y Vanous ${ }^{18}$ precisan que el precio del petróleo de 1975 era determinado como media móvil sobre tres años en el periodo 1972-74, para 1976 se tomó el periodo 1972-75 y a partir de 1977 se aplicó la nueva fórmula con la media móvil calculada sobre el precio del mercado mundial de los cinco años anteriores. Según otros analistas «el acuerdo comercial de 1976 entre Cuba y la URSS establecía que Cuba comprara el petróleo de la URSS al precio promedio del mercado mundial de los 5 años anteriores $\rangle^{19}$ pero no precisan la nueva fórmula utilizada.

Como mostraremos en este trabajo, al margen de los acuerdos firmados, los soviéticos favorecieron al gobierno revolucionario cubano no solo a través de créditos y precios preferenciales, sino también concediendo márgenes de maniobra no convencionales en las prácticas comerciales que resultaron muy ventajosos para la isla. Empezamos aclarando las ganancias para Cuba de las reexportaciones de azúcar y petróleo, y las expresamos construyendo indicadores que muestran su impacto sobre la economía cubana. Sucesivamente analizamos el comercio entre Cuba y la ex Unión Soviética, evidenciando como las reexportaciones de petróleo se contabilizaban como exportaciones a la URSS, probablemente para aparentar un menor déficit comercial con este país. Demostramos que una parte del petróleo importado de la ex URSS venía reexportado a países del área capitalista, para generar ingresos en divisa, por tanto, no es correcto contabilizarlo como una exportación a la Unión Soviética, como hicieron las autoridades cubanas, según un examen cuidadoso del intercambio entre los dos países con datos de los anuarios estadísticos de Cuba (AEC). Con esta revisión contable — restando a las exportaciones cubanas a la URSS las reexportaciones de petróleo- procedemos a medir el subsidio soviético a la economía cubana rechazando la medición en

\footnotetext{
7 Pérez López, 1979: 290.

${ }^{18}$ Marrese y Vanous, 1983: 66.

19 Zimbalist y Brundenius, 1989: 152.
} 
dólares utilizada en todos los trabajos hasta ahora, y proponiendo un método original con la finalidad de mostrar sus verdaderas repercusiones sobre la economía de la isla.

\section{LAS REEXPORTACIONES DE AZÚCAR}

En la década de los ochenta, el comercio entre Cuba y la URSS ${ }^{20}$ incluye las reexportaciones por parte de la isla de los dos principales productos en el intercambio entre los dos países: el azúcar y el petróleo. Consideramos que en la literatura el impacto sobre la economía cubana de este comercio se ha subestimado o medido de manera poco correcta, sin permitir entender su real importancia para el país caribeño. A continuación, tratamos de subsanar este problema.

El AEC reporta entre las exportaciones cubanas las reexportaciones de azúcar y petróleo, siendo productos que la isla compraba para venderlos a terceros países, «sin atravesar las fronteras nacionales». Es decir, Cuba importaba azúcar del mercado libre y petróleo de la URSS, que vendía a otros países y los incluía entre las exportaciones ${ }^{21}$.

En relación a las reexportaciones de azúcar, las informaciones oficiales provienen de los boletines económicos del Banco Nacional de Cuba (BNC) que dan solo datos en valor (en pesos cubanos) mientras la misma fuente aclara que Cuba compraba el azúcar en el mercado libre y lo pagaba en moneda convertible (dólares estadunidenses). Para estimar las cantidades reexportadas, lo que tratan de hacer por ejemplo Ritter y Pérez López ${ }^{22}$, es necesario transformar en dólares los datos del BNC, utilizando la tasa oficial de cambio y luego dividir entre el precio del azúcar en el mercado mundial. Aplicando este procedimiento para la década de los ochenta, obtenemos los siguientes resultados:

${ }^{20}$ Un análisis reciente del sector exterior cubano en la primera parte del periodo revolucionario (hasta la caída del muro de Berlín) que evidencia sus peculiaridades a partir de la concentración de los intercambios comerciales con la ex Unión Soviética, se encuentra en García Fernández y Sovilla (2017).

${ }^{21}$ Anuario Estadístico de Cuba, 1989: 245.

22 Ritter, 1990: 147. Pérez López, 1991: 161. 
TABla 1. ReEXPortaciones de AZÚCAR

\begin{tabular}{|c|c|c|c|}
\hline \multicolumn{2}{|c|}{$\begin{array}{c}\text { Ingresos } \\
\text { (Millones de pesos) }\end{array}$} & $\begin{array}{c}\text { Cantidades } \\
\text { (Millones de toneladas) }\end{array}$ & $\begin{array}{c}\text { Reexportaciones/ exportaciones } \\
\text { totales de azúcar (\%) }\end{array}$ \\
\hline 1982 & 16,4 & 0,11 & 1,37 \\
\hline 1983 & 38,6 & 0,24 & 3,42 \\
\hline 1984 & 101 & 1,00 & 14,2 \\
\hline 1985 & 106,4 & 1,3 & 18,02 \\
\hline 1986 & 89,6 & 0,81 & 12,14 \\
\hline 1987 & 189,4 & 1,27 & 19,62 \\
\hline 1988 & 199,8 & 0,89 & 12,77 \\
\hline 1989 & 345 & 1,22 & 17,16 \\
\hline
\end{tabular}

Fuente: para las importaciones de azúcar de 1982-83: Ritter (1990: 147); para el periodo 1984-89: (Banco Nacional de Cuba, 1986: 28; 1987: 25; 1988: 16; 1989: 12; 1990: 23). Para las exportaciones totales de azúcar en 1982: Anuario Estadístico de Cuba (1985: 432); en 1983-88: Anuario Estadístico de Cuba (1988: 466-67); en 1989: Rodríguez (1992b: 11).

Citando la misma fuente (informes anuales del BNC) ${ }^{23}$, Pérez López estima las reexportaciones de azúcar en 670.000 toneladas en 1986 y 1.270 .000 toneladas en 1987. El segundo dato es igual al que estimamos para ese año, pero el primero es bastante inferior. El valor estimado por Pérez López para 1986 sería coherente con un tipo de cambio entre peso cubano y dólar de 1:1, lo cual es cierto solo a partir de 1987, mientras que en 1986 dicha tasa era de 1,21 dólares por 1 peso cubano ${ }^{24}$. Por tanto al tipo de cambio oficial vigente en 1986, las reexportaciones en ese año son de 812.840 toneladas y no de 670.000 como afirma el investigador cubano-americano.

La última columna de la tabla 1 se construyó para mostrar la relevancia de estas reexportaciones, poniéndolas en relación con las exportaciones de azúcar totales y alcanzando un valor máximo de casi el $20 \%$ en 1987.

¿Cómo determinar con mayor precisión el impacto para la economía cubana de estas reexportaciones? Contestar a esta pregunta es un problema de difícil solución, dada la dificultad que comporta el comparar datos en moneda fuerte (dólares norteamericanos), con otros en moneda convenio (rublos no convertibles), moneda utilizada en las transacciones entre Cuba y los países del CAME, destacando entre ellos la URSS. Se trata de dos tipologías de comercio distintas: la primera es la modalidad típica de los países capitalistas, que utilizan la moneda de reserva convertible (el dólar norteamericano); la segunda, la de los países del CAME, que utilizan como medio de pago el

${ }^{23}$ Pérez López, 1991: 161.

24 Banco Nacional de Cuba, 1987: 47. 
rublo soviético, no convertible, que se denominaba moneda convenio. Académicos especializados en Cuba, de reconocido nivel, han incurrido en graves incongruencias en el análisis del comercio exterior cubano, por querer sumar los dos tipos de comercio, el que se expresaba en moneda convertible y el otro en moneda convenio dentro del CAME.

Por ejemplo, los trabajos de Ritter y Pérez López padecen los problemas señalados y, a pesar de reconocer que las reexportaciones de azúcar representan una actividad que aporta a Cuba enormes ganancias, no las pueden medir de manera correcta. Según Pérez López ${ }^{25}$ : «El BNC reportó que en 1984-85 Cuba compró azúcar en el mercado mundial por un valor de 201 millones de pesos; reexportado a la URSS (también Pérez López asume que las ventas a los países socialistas, en prevalencia a la URSS sean en realidad todas a la URSS) generó 1.529 millones de pesos por una ganancia neta de 1.328 millones de pesos o un rendimiento de la inversión de $661 \%{ }^{26}$. De la misma manera, Ritter $^{27}$ calcula entre 1982-87 ganancias de 3.497,3 millones de pesos, mayores que todas las exportaciones a la URSS en 1982 (3.289 millones de pesos). En otra intervención ${ }^{28}$ el autor reconoce que

... comprando [el azúcar] al precio del mercado libre y vendiéndolo a la Unión Soviética $^{29}$ al precio f.o.b., Cuba tenía una ganancia entre 750 y 1.300 millones de pesos en esos años. Sin embargo, el tipo de cambio no es realista y las ganancias en rublos se tienen que gastar en importaciones de la URSS y de los otros países del CAME (cuyas exportaciones a menudo no son competitivas en precio y calidad)... las estimaciones de las ganancias por reexportación de azúcar han sido probablemente excesivas.

25 Pérez López, 1991: 161.

${ }^{26}$ Banco Nacional de Cuba, 1986: 25. La cursiva es nuestra.

27 Ritter, 1990: 147.

${ }^{28}$ Ritter, 1988: 126.

${ }^{29}$ Es lógico que las reexportaciones de azúcar se dirigieran hacia la zona del CAME, y no a los países capitalistas. Hay que descartar la posibilidad de que Cuba comprara azúcar del mercado libre para venderlo a los países capitalistas, y mantener las entregas prometidas, dada su poca importancia en las exportaciones totales de azúcar, que Cuba garantizaba sin dificultad en aquellos años. Otra posibilidad que me expresó Pérez López en una carta privada, es que Cuba comprara el azúcar en el mercado mundial para ahorrar en los costos de transporte: «Es posible que la empresa cubana para la exportación de azúcar (Cubazucar) se hubiera dado cuenta que era más conveniente comprar azúcar en Tailandia o en las Philipinas, y llevar ese azúcar hasta Australia, en lugar de transportar el azúcar cubano hasta Australia». Esta hipótesis me parece equivocada, porque un informe del Banco Nacional de Cuba (1986: 25) aclara que el azúcar era reexportado «a los países socialistas» Ese mismo informe del Banco Nacional de Cuba precisa que estas reexportaciones eran dirigidas «a los países socialistas, en particular a la URSS», y como el $80 \%$ de las exportaciones cubanas al campo socialista iban a la URSS, yo supuse que tuvieran como único destino este país. Pérez López hace la misma hipótesis (1991: 161 ), entrando en contradicción con la que me sugirió en el intercambio epistolar señalado. 
Los razonamientos de Ritter y Pérez López merecen una reflexión, pues permiten individuar algunos importantes problemas, que como veremos más adelante se vuelven a presentar en la medición del subsidio. Como los mismos autores reconocen, el tipo de cambio oficial no tiene significado económico ${ }^{30}$, por tanto no se puede utilizar con tanta superficialidad agregando datos en dólares (moneda convertible) y rublos (moneda convenio). Además, a menudo, en el análisis de problemas específicos como esto de las reexportaciones de azúcar, es posible llegar a conclusiones conceptualmente validas sin tener que pasar necesariamente por la tasa de cambio oficial, para sumar datos en moneda fuerte y moneda convenio.

En efecto, los autores hubiesen podido razonar de la siguiente manera para medir los beneficios de la economía cubana derivados de la compra de azúcar en el mercado mundial: 1) considerar ese gasto en dólares y medir su poder de compra en términos de petróleo dividiéndolo entre su precio en el mercado mundial; 2) multiplicar la cantidad de azúcar que Cuba compraba en el mercado mundial, que se determina dividiendo el gasto para comprar azúcar entre su precio en el mercado mundial, por el precio pagado por la URSS, calculando los ingresos para Cuba en moneda convenio; 3 ) dividir este valor entre el precio del petróleo pagado a la URSS (también medido en moneda convenio), determinando la cantidad de petróleo que Cuba recibía de la URSS como contrapartida del azúcar que compraba en el mercado mundial; 4) la diferencia entre la cantidad de petróleo resultante en el punto 3) con la del punto 1) multiplicada por el precio del petróleo en el mercado mundial, permite estimar la ganancia para Cuba, en moneda convertible, por esta actividad de comercialización del azúcar (compra en el mercado mundial para vender a la URSS).

La tabla 2 resume los resultados.

TABLA 2. IngRESOS ESTIMADOS EN DÓLARES ESTADUNIDENSES POR LAS REEXPORTACIONES

DE AZÚCAR SOBRE EL TOTAL DE LAS EXPORTACIONES EN MONEDA CONVERTIBLE

\begin{tabular}{|c|c|}
\hline 1982 & $4,2 \%$ \\
\hline 1983 & $20,9 \%$ \\
\hline 1984 & $104,2 \%$ \\
\hline 1985 & $149,7 \%$ \\
\hline 1986 & $61,4 \%$ \\
\hline 1987 & $116,9 \%$ \\
\hline 1988 & $45,8 \%$ \\
\hline
\end{tabular}

Fuente: para la estimación de las reexportaciones de azúcar véase tabla 1. Para los ingresos en moneda convertible: Banco Nacional de Cuba (1986: 23) para el periodo 1982-85; Banco Nacional de Cuba (1987: 23) para 1986; Banco Nacional de Cuba (1988:15) para 1987; Banco Nacional de Cuba (1989: 15) para 1988; Banco Nacional de Cuba (1990: 22) para 1989.

30 Pérez López, 1992: 32. 
La tabla 2 nos da una idea de la importancia para Cuba de la reexportación del azúcar comprado en el mercado mundial. En tres años $(1984,1985,1987)$ esa ganancia estimada es mayor que el valor de todas las exportaciones en moneda convertible a los países capitalistas (al neto de las reexportaciones de petróleo $)^{31}$ : en el periodo $1982-89$ equivale en promedio a $2 / 3$ de estas mismas exportaciones.

Hemos propuesto un ejercicio de estimación de los beneficios para el gobierno cubano por la reexportación de azúcar a partir de un método que nos parece más coherente que los ejercicios realizados hasta ahora, sin embargo, no hay que olvidar que Cuba compraba el azúcar en el mercado mundial porque no lograba garantizar a los países del CAME las entregas pactadas con su propia producción interna.

\section{LAS REEXPORTACIONES DE PETRÓLEO}

Los informes anuales del Banco Nacional de Cuba (BNC) entre 1985 y 1990, reportan que Cuba re-exportaba parte del petróleo importado de la URSS para obtener moneda libremente convertible ${ }^{32}$. Mesa Lago (2000: 243) confirma que la Unión Soviética «hizo otra importante concesión: pagarle a Cuba en moneda convertible por el petróleo que se había comprometido a entregar y que la isla podía ahorrar; la Unión Soviética exportaba ese petróleo a otros países y le pasaba a Cuba los ingresos, que esta contabilizaba como reexportaciones de petróleo». Según Pérez López ${ }^{33}$.

La mecánica de estas reexportaciones no era muy clara. Considerando que las exportaciones de petróleo soviético a Cuba tenían que viajar por 6.500 millas del Mar Negro hasta los puertos cubanos, el hecho de volver a exportar hasta Europa desde Cuba el petróleo no sería económico. Parece más probable que los barcos, con el crudo soviético fueran enviados directamente de la URSS por encargo de Cuba, a los compradores europeos. Eso sería consistente con la referencia que se hace, en la literatura cubana, al petróleo como exportaciones a la URSS y explicaría porque las estadísticas sobre las importaciones por parte de los probables compradores de estas reexportaciones no registran totalmente los volúmenes anticipados. Por ejemplo las

${ }^{31}$ A continuación, se explicará porque las reexportaciones de petróleo no se incluyen entre las exportaciones a la URSS.

${ }^{32}$ Los informes del BNC dan los datos en pesos cubanos, pero aclaran que Cuba ingresaba moneda convertible (dólares), que en los mismos informes se transforman en pesos a la tasa oficial de cambio.

33 Pérez López, 1986: 28. 
estadísticas de las importaciones por parte de los países de Europa Occidental... reportan importaciones de Cuba por 85 millones de dólares (alrededor de 71 millones de pesos a la tasa de cambio oficial) en 1982, 113 millones de dólares (97 millones de pesos) en 1983, y 50 millones de dólares (44 millones de pesos) en 1984, mientras las fuentes cubanas registran exportaciones aproximadamente por 5 veces ese valor en 1982, 6 veces en 1983, y 12 veces en 1984 .

Esto significa que la URSS vendía el petróleo a los países occidentales y por esta razón no lo registraba como importación desde Cuba que, sin embargo, se beneficiaba de los ingresos en moneda fuerte.

El gobierno cubano y algunos estudiosos ${ }^{34}$, consideraban que estas reexportaciones eran posibles por dos razones: 1) la exitosa política de ahorro energético, «el consumo energético por cada peso de producto social global $(\mathrm{PSG})^{35}$ se redujo el $25 \%$ entre $1980-85 »^{36}$ y la sustitución del petróleo con bagazo para alimentar los centrales azucareros; 2) el aumento de la producción interna de petróleo que alcanzó las 867.000 toneladas en 1985 (más que triplicando la producción de 1981).

Estas observaciones son correctas, pero olvidan el factor más importante, es decir, el gran incremento de las entregas soviéticas de petróleo, de 9-10 millones de toneladas anuales a finales de los setenta, hasta más de 13 millones después de 1983, cuando las reexportaciones de petróleo empezaron a ser importantes. Fue gracias al aumento de los suministros soviéticos que Cuba podía continuar a consumir casi 10 millones de toneladas de crudo, también después de 1983 cuando las reexportaciones fueron alrededor de 3 millones de toneladas anuales.

Con eso no queremos minimizar los progresos en cuanto a un uso más racional de los recursos energéticos, en el marco de una mayor eficiencia con la implementación del sistema de dirección y planificación de la economía (SDPE) a partir de $1980^{37}$.

Sin embargo lo que importa subrayar en este caso es que la referencia que hace Pérez López a la literatura cubana ${ }^{38}$, que menciona las reexportaciones

${ }^{34}$ Zimbalist y Brundenius, 1989: 145.

${ }^{35}$ El PSG era el principal indicador productivo utilizado en la contabilidad nacional de las economías planificadas. No tomaba en cuenta los servicios no materiales, y no se medía con el método del valor agregado, diferenciándose así del producto interno bruto (PIB) que se utiliza en los países capitalistas.

${ }^{36}$ Banco Nacional de Cuba, 1986: 5.

37 Sovilla y García Fernández, 2013: 169.

38 Este autor no da indicaciones sobre las fuentes, sin embargo, una de ellas es Rodríguez (1992b: 4), que mencionando las exportaciones cubanas a la URSS afirma que «no mostraron variaciones sustanciales [...] absorbiendo fundamentalmente las de azúcar y de níquel [...] también se reexportó petróleo soviético ahorrado por parte de Cuba desde 1977, lo cual apor- 
de petróleo a la URSS, se confirma plenamente en nuestro análisis de los datos del Anuario Estadístico de Cuba (AEC) de las exportaciones cubanas a los países capitalistas y a la misma URSS.

En la tabla 3, a partir de datos oficiales del AEC, construimos la relación entre la suma de todas las exportaciones reportadas en el anuario ${ }^{39}$ a la URSS y el total de ellas, en el periodo 1976-88.

TABla 3. REEXPORTACIONES DE PETRÓLEO Y OtRAS EXPORTACIONES A LA URSS

\begin{tabular}{|c|c|c|c|}
\hline Año & $(1)$ & $(2)$ & $(3)=(2)+(1)$ \\
\hline 1976 & $99,82 \%$ & & $99,82 \%$ \\
\hline 1977 & $97,85 \%$ & & $97,85 \%$ \\
\hline 1978 & $99,15 \%$ & & $99,15 \%$ \\
\hline 1979 & $99,48 \%$ & & $99,48 \%$ \\
\hline 1980 & $95,57 \%$ & $4,44 \%$ & $100,01 \%$ \\
\hline 1981 & $92,88 \%$ & $6,42 \%$ & $99,30 \%$ \\
\hline 1982 & $91,76 \%$ & $7,85 \%$ & $99,61 \%$ \\
\hline 1983 & $87,23 \%$ & $12,82 \%$ & $100,05 \%$ \\
\hline 1984 & $87,29 \%$ & $12,26 \%$ & $99,55 \%$ \\
\hline 1985 & $88,40 \%$ & $11,76 \%$ & $100,16 \%$ \\
\hline 1986 & $93,21 \%$ & $6,31 \%$ & $99,52 \%$ \\
\hline 1987 & $91,03 \%$ & $7,40 \%$ & $98,43 \%$ \\
\hline 1988 & $92,87 \%$ & $5,02 \%$ & $97,89 \%$ \\
\hline
\end{tabular}

(1): Exportaciones a la URSS registradas en los AEC divididas entre el total de las exportaciones a la URSS.

(2): Reexportaciones de petróleo a la URSS divididas entre el total de las exportaciones a la URSS.

Fuente: Anuario Estadístico de Cuba (1981: 208-212) para el total de las exportaciones a la URSS y la suma de las que aparecen en los anuarios, en el periodo 1976-80; Anuario Estadístico de Cuba (1985: 430-441) para 1981-82; Anuario Estadístico de Cuba (1988: 464-474) para el periodo 1983-1988. Para las reexportaciones de petróleo: Anuario Estadístico de Cuba (1985: 400) para 1980; Banco Nacional de Cuba (1986: 23) para 1981-85; Banco Nacional de Cuba (1987: 23) para 1986; Banco Nacional de Cuba (1989: 15) para 1987-88; Banco Nacional de Cuba (1990: 22) para 1989.

tó mayores ingresos al país estimables en 3.000 millones de pesos en moneda convertible hasta 1989», concepto que el autor reiteró (Rodríguez, 1992a: 55).

39 Además de azúcar, níquel y cítricos, Cuba exportaba pocos productos más, entre ellos bebidas alcohólicas, tabaco, cigarrillos. 
Entre 1976 y 1979 la columna (1) es muy cercana a la unidad, ya que se encontraba entre el 97,9\% y 99,8\%, lo cual significa que ninguna otra exportación a la URSS, además de las registradas en el AEC, existió en aquellos años. De repente, la relación cayó hasta 95,6 \% en 1980 y siguió disminuyendo en los siguientes años llegando a su valor mínimo de $87,2 \%$ y $87,3 \%$ entre 1983-84.

Es difícil creer que entre 1983-85, por ejemplo, hayan surgido nuevas exportaciones a la URSS por un valor del $12-13 \%$ de las exportaciones totales sin que resultaran registradas en las estadísticas oficiales cubanas y en ninguna publicación de investigadores cubanos y extranjeros. Sin embargo, si consideramos las reexportaciones de petróleo y las sumamos a las otras registradas, obtenemos la columna 3 de la tabla 2. Los resultados dejan pocas dudas: la relación entre exportaciones registradas y totales regresa a los valores de los años setenta, entre $99 \%$ y $100 \%$ en el periodo 1980-86 y cae ligeramente en $1987(97,9 \%)$, año en el cual Cuba comenzó a exportar productos electrónicos y farmacéuticos ${ }^{40}$, dos agregados que empiezan a ser importantes entre 1988-9041.

En conclusión: una parte del petróleo que Cuba importaba de la URSS en los ochenta, se vendía a los países occidentales para generar ingresos en moneda convertible y, lo más interesante, es que los anuarios estadísticos de Cuba las reportaban como exportaciones a la URSS.

Hay otros elementos que confirman nuestra hipótesis: los informes del BNC muestran en 1981 exportaciones en moneda convertible mayores que las exportaciones a los países capitalistas, lo cual indica que había exportaciones en moneda convertible hacia los países socialistas ${ }^{42}$. La posibilidad de que estas exportaciones a los países socialistas en moneda convertible fueran compuestas por azúcar debe ser descartada, pues como vimos en el párrafo anterior, desde 1982 Cuba tuvo que comprar azúcar en el mercado libre para mantener las entregas pactadas a los países socialistas, pagadas en moneda convenio. Si quitamos de las exportaciones totales en moneda convertible las reexportaciones de petróleo registradas por el $\mathrm{BNC}$, las exportaciones a los países capitalistas y en moneda convertible casi coinciden (a pesar de la distinta proveniencia de los datos $)^{43}$, con la excepción de 1981-82, lo cual im-

${ }^{40}$ Cabe observar que inmediatamente fueron detectadas por los investigadores cubanos, aun siendo muy inferiores a las que fueron ignoradas en 1980.

${ }^{41}$ Rodríguez, 1992b: 9.

42 En las estadísticas cubanas se diferenciaba entre países capitalistas y socialistas, estos últimos eran los países miembros del CAME y China.

${ }^{43}$ Las exportaciones en moneda convertible provienen de los informes del BNC, y las exportaciones a los países capitalistas de los AEC. 
plica que las exportaciones en moneda fuerte a los países socialistas eran las reexportaciones de petróleo.

Dulcis in fundo, podemos entender el dato de la deuda cubana hacia la URSS del entonces jefe de gobierno soviético Ryzkhov que lo fijaba en 1990 en 15.000 millones de rublos, mientras que los economistas cubanos lo estimaban en 13.800 millones de pesos ${ }^{44}$. Si asumimos que los cubanos incluyeran en las exportaciones a la URSS el petróleo reexportado y los soviéticos no lo consideraran entre las importaciones provenientes de Cuba, siendo petróleo que ellos mismos le exportaban, la cifra de Ryzkhov que corresponde a 16,6 billones de pesos casi coincide con la de Rodríguez.

En resumen: hay una serie de pruebas indirectas, pero ninguna admisión oficial, de que las estadísticas cubanas incluyeran entre las exportaciones a la URSS el petróleo que Cuba vendía a los países capitalistas europeos en dólares. 1) Un cuidadoso examen de las estadísticas oficiales cubanas muestra que, desde 1976, el AEC registró todas las exportaciones a la URSS, pero a partir de 1980 aparece un vacío que aumenta después de 1983 y que se explica perfectamente si consideramos las reexportaciones de petróleo entre las exportaciones a la URSS; 2) Si comparamos el total de las exportaciones en moneda convertible a los países capitalistas con el total de las exportaciones en moneda convertible (datos que provienen de distintas fuentes oficiales cubanas), observamos que las reexportaciones de petróleo se veían contabilizadas entre las exportaciones en moneda convertible pero no a los países capitalistas, por tanto debían dirigirse al campo socialista; 3) en al menos dos ocasiones el destacado economista cubano José Luis Rodríguez ${ }^{45}$, experto conocedor de las relaciones comerciales de la isla, trató las reexportaciones de petróleo como exportaciones a la URSS ${ }^{46}$; 4) la contabilización de las reexportaciones de petróleo como exportaciones a la URSS permite explicar perfectamente las distintas cifras entregadas por investigadores cubanos y políticos soviéticos sobre la deuda cubana hacia la URSS.

Entre 1984 y 1986, las reexportaciones de petróleo permitieron al gobierno revolucionario cubano de mantener las importaciones de los países capitalistas al doble del valor de las exportaciones $\mathrm{y}$, al mismo tiempo, poder negociar con éxito el pago de la deuda. Esto ocurrió con el precio del azúcar en constante caída, y fue posible por el permiso de la URSS para que Cuba

44 Rodríguez, 1993: 7.

45 Pérez López también cita «fuentes cubanas» que ponen las reexportaciones de petróleo entre las exportaciones a la URSS, sin identificarlas. Con toda probabilidad se trata de los trabajos citados de Rodríguez.

46 Rodríguez, 1992b: 4; 1992a: 55. 
pudiera reexportar hacia los países occidentales una parte del petróleo que le vendía y aumentar así los ingresos en moneda convertible.

Para dar una idea más concreta de la importancia de estos ingresos, construimos la tabla 4 donde sumamos el valor de las exportaciones de azúcar en el mercado mundial en el quinquenio 1981-85 y las reexportaciones de petróleo (ambas significaban ingresos en moneda convertible), dividiendo el resultado entre la cantidad de azúcar que Cuba exportó en aquellos años en el mercado mundial, llegando así a expresar, en términos del precio del azúcar, lo que significó la autorización de la URSS a reexportar petróleo.

Tabla 4: Precio real y virtual del azúcar (Centavos de dólar por libra)

\begin{tabular}{|c|c|c|}
\hline Año & Precio real & Precio virtual $(*)$ \\
\hline 1981 & 16,83 & 26,3 \\
\hline 1982 & 8,35 & 32,1 \\
\hline 1983 & 8,49 & 24,9 \\
\hline 1984 & 5,2 & 25,8 \\
\hline 1985 & 4,06 & 19,6 \\
\hline
\end{tabular}

(*) Explicación en el texto.

Fuente: Banco Nacional de Cuba (1986: 23) y Fernández Font (1989: 111, 197).

El resultado nos parece interesante e indicativo. Para Cuba, reexportar petróleo en aquellos años era equivalente a mantener los niveles excepcionales del precio del azúcar de $1980^{47}$, en un periodo en el cual este precio había regresado a los niveles muy bajos de los años sesenta (4-5 centavos por libra en 1985). Entre 1981 y 1985 las reexportaciones de petróleo significaron un ingreso de 2.185 millones de dólares (alrededor de 2.000 millones de pesos), un $30 \%$ más que los egresos por pago de intereses sobre la deuda externa en moneda libremente convertible que, en el mismo periodo, fueron de 1.436 millones de pesos. Dichas reexportaciones, entre 1983-85, representaron la primera fuente de ingreso en moneda convertible para el gobierno cubano.

47 A la misma conclusión llegan Domínguez (1989: 101-102) y Pérez López (1991: 227), según el cual las reexportaciones de petróleo permitieron «compensar la caída de moneda convertible por los precios bajos del azúcar en el mercado mundial». 


\section{El subsidio SOVIÉTICO}

Lo que hemos discutido en las secciones anteriores nos permite ahora pasar al tema central de este trabajo, es decir, la medición del subsidio soviético a Cuba.

El objetivo de esta investigación es individuar y cuantificar el flujo de recursos que llegó a la economía cubana a través de las relaciones económicas preferenciales con la URSS. Siguiendo las indicaciones de Rodríguez, quien propone separar los créditos obtenidos de los países del CAME de los que provenían de bancos comerciales occidentales porque «sus repercusiones dentro de la economía cubana eran totalmente distintos ${ }^{48}$, mantendré separadas las transacciones reales y financieras en rublos transferibles (o moneda convenio) de las transacciones en moneda libremente convertible. Esto para definir de la manera más precisa y concreta posible los beneficios para la economía cubana derivados de las condiciones preferenciales en el comercio que le otorgaron los soviéticos.

Para aclarar las relaciones económicas entre los dos países, seguiré los siguientes pasos: a) efectuaré un ajuste contable en los datos oficiales de los AEC, sustrayendo de las exportaciones cubanas a la URSS las reexportaciones de petróleo; b) mostraré todas las fuentes de financiamiento provenientes de la URSS a la economía cubana, separando las que eran en moneda fuerte (dólares y otras divisas convertibles) y convenio (rublos no convertibles); c) construiré indicadores conceptualmente válidos, prácticos y funcionales, o sea capaces de dar una visión concreta de la ayuda soviética midiendo su impacto sobre la economía cubana, sin caer en el error de transformar en moneda fuerte datos expresados en moneda convenio o viceversa, usando el tipo de cambio oficial para la conversión.

\section{3a. Conexión con la literatura}

El primer intento de medir el subsidio soviético a Cuba fue efectuado por la CIA (1976), y como observaron Zimbalist y Brundenius ${ }^{49}$ : «la mayor parte de los cubanólogos se basa sobre las estimaciones de la ayuda soviética entregadas por la CIA». En el trabajo citado, la CIA trata de individuar las distintas formas de subsidio, agregarlas y, en un estudio siguiente (1986), mejorar las estimaciones, considerando que el sistema de los precios prefe-

\footnotetext{
48 Rodríguez, 1985: 100.

49 Zimbalist y Brundenius, 1989: 150.
} 
renciales no implicaba solo un subsidio a las exportaciones de azúcar ${ }^{50}$ sino también a las de níquel y a las importaciones de petróleo. Mesa Lago (2000: 258) cuantifica «la ayuda soviética total a Cuba, combinando préstamos y subsidios por precios (al azúcar, al níquel, al petróleo, todos basados en los precios del mercado mundial) pero excluyendo los ingresos por reexportaciones, en 39.8 billones de dólares norteamericanos entre 1971 y 1985».

En sus estimaciones iniciales, la CIA detectaba dos componentes del subsidio: los créditos comerciales y los precios preferenciales (llamado subsidio implícito) al azúcar cubano. Para poderlas agregar utilizaba el tipo de cambio oficial peso/dólar y expresaba en dólares el subsidio total que es la suma de los déficit comerciales y del componente implícito del subsidio, calculado multiplicando la diferencia entre el precio pagado por la URSS (transformado en dólares al tipo de cambio oficial) y el precio del mercado mundial por la cantidad de azúcar que Cuba exportaba a la Unión Soviética.

El trabajo de la CIA ha sido criticado por Radell ${ }^{51}$, quien introdujo dos ajustes: 1) en el cálculo del subsidio al precio del azúcar el autor compara el precio preferencial pagado por los soviéticos con el precio también preferencial que pagaban los norteamericanos, y no con el precio más bajo del mercado mundial (como en las estimaciones de la CIA); 2) considera el costo adicional de los productos importados de la URSS con respecto a sus precios en el mercado mundial. No obstante a lo largo de su trabajo Radell mencione el problema principal, o sea, que «los soviéticos no pagan normalmente en moneda libremente convertible $»^{52}$, y los pagos eran en rublos transferibles utilizables solo dentro del CAME, no lo toma en cuenta y su estimación es muy parecida a las de la CIA.

Siguiendo el mismo razonamiento, Zimbalist y Brundenius ${ }^{53}$ también critican el trabajo de la CIA, afirmando:

Para estimar este subsidio la CIA 1) usa la tasa de cambio oficial peso/dólar, 2) ignora el aspecto tied del subsidio, es decir el hecho que los pagos son casi totalmente créditos en rublos que se pueden usar solo para comprar productos soviéticos de calidad inferior, y 3) mide el subsidio como diferencia entre el precio convertido en dólares pagado por los soviéticos, y el precio del mercado libre. Los incisos 1) y 2) no tienen justificación económica y causan una significativa distorsión hacia arriba. El tercer punto es arbitrario y muestra o un perjuicio político, o una comprensión equivocada del mercado mundial del azúcar.

\footnotetext{
${ }^{50}$ Mesa Lago y Gil (1988: 67) incluyen otras dos importaciones: trigo y maíz.

${ }^{51}$ Radell, 1983: 377.

52 Ibidem: 371.

${ }^{53}$ Zimbalist y Brundenius, 1989: 150.
} 
Estas observaciones son sin duda oportunas como veremos, sin embargo, sorprende que los dos expertos sobre Cuba dediquen poco espacio a este análisis (solo 4 páginas de su extenso trabajo) y procedan, después de haber señalado estos problemas, por afirmaciones parciales ${ }^{54}$ que no ayudan a entender las complejas relaciones comerciales entre los dos países y, lo que más importa, sin proponer un método alternativo para estimar el subsidio soviético. Zimbalist y Brundenius parecen darse cuenta del grave error metodológico de las estimaciones de la CIA que comentaré a continuación, sin embargo, al final, comparando el subsidio soviético a Cuba al que recibió Puerto Rico de los Estados Unidos, vuelven a tomar en cuenta, para medir el primero, las estimaciones de la CIA, ignorando los problemas que ellos mismos señalan y que no justifican dicha comparación.

También Turits ${ }^{55}$ rechaza las estimaciones de la CIA, afirmando que cuanto más se pueden considerar «el nivel superior para la estimación del subsidio soviético». Desde otra perspectiva, el autor hace hincapié en los mismos problemas señalados por Zimbalist y Brundenius, siendo el más importante «la hipótesis extrema que la característica "tied" del subsidio soviético no implique costos de oportunidad significativos», que lleva a la CIA a estimar el subsidio como diferencia «entre los valores en el mercado mundial de las exportaciones de azúcar cubano y las importaciones de petróleo, y los montos efectivamente pagados» ${ }^{56}$. Según Turits:

El problema más directo de las estimaciones de la CIA es la afirmación no demostrada que el trato favorable que Cuba obtuvo en el intercambio azúcar/petróleo, no sea reducido por la selección y los términos de intercambio con los otros $\operatorname{productos}^{57}$.

El autor añade, además:

En efecto los productos petroleros constituyen solo alrededor de un tercio de todas las importaciones cubanas de la URSS. Una cantidad casi equivalente son las maquinarias y equipo de transporte, y los productos soviéticos no se consideran nor-

${ }^{54}$ Así Zimbalist y Brundenius (1989: 152) mencionan el empeoramiento para Cuba de los términos de intercambio entre 1980-85, el mayor costo de los productos soviéticos y su baja calidad; ninguno de estos problemas viene analizado ni considerado en un nuevo intento de medición del subsidio que tome en cuenta su característica «tied» y el problema del tipo de cambio. Por el contrario, los mismos autores aceptan la tasa de cambio oficial cuando afirman que los productos soviéticos eran más caros y que en algunos años (desde 1986) Cuba pagaba más que el precio del mercado mundial para el crudo soviético (idem).

55 Turits, 1987: 174-175.

${ }^{56}$ Idem.

${ }^{57}$ Idem. 
malmente de la mayor calidad...los cálculos del subsidio usan las tasas de cambio oficiales, que pueden ser equivocadas, ya que el peso no es una moneda convertible sujeta a las fuerzas del mercado, siendo establecida por el gobierno. Con una cotización en el mercado negro de 5-6 pesos por un dólar, comparada con la oficial de 0,9 pesos por un dólar, la cotización oficial del peso parece apreciarlo con respecto al dólar ${ }^{58}$.

Turits se da cuenta que «el problema es que las estimaciones de la CIA de la ayuda soviética a Cuba han sido aceptadas por muchos autores sin una discusión de los problemas metodológicos anexos» ${ }^{59}$ y que no es correcto comparar Cuba con otros países con economía de mercado (por ejemplo Israel) en términos del subsidio per cápita recibido, sin embargo, como Zimbalyst y Brundenius, no propone un método alternativo de estimación de la ayuda soviética a Cuba, por el contrario, observa que

... un cálculo preciso del subsidio soviético implícito a Cuba obligaría a construir un modelo económico para los términos de intercambio en su conjunto, las cuentas del sector externo y las tasas de cambio derivadas, lo cual va más allá del objetivo de este artículo ${ }^{60}$.

Por tanto, también en este caso, la crítica de los métodos tradicionales de estimación parece genéricamente dirigirse a reducir la importancia del subsidio soviético a Cuba, en lugar de buscar otros métodos que superen los problemas correctamente detectados.

En la literatura, otro interesante intento de estimar el subsidio soviético es el de Ritter. En sus palabras: «la medición de la asistencia económica es difícil e imprecisa, tanto conceptualmente como prácticamente, pero estimaciones razonables de algunas partes de la ayuda se pueden hacer» ${ }^{61}$. Después de una clara definición de lo que reconoce ser un subsidio, o sea «el valor de los varios componentes de la relación especial entre Cuba y la URSS, respecto a lo que hubiese sido su valor si Cuba hubiese participado de la misma manera y en la misma medida en el mercado mundial ${ }^{62}$, Ritter distingue dos secciones: 1) los créditos comerciales y al desarrollo ${ }^{63}$ y 2) el subsidio implícito a través de precios preferenciales a las exportaciones e importaciones.

A partir de la siguiente consideración:
58 Idem.
${ }^{59}$ Idem.
${ }^{60}$ Idem.
${ }^{61}$ Ritter, 1990: 124.
62 Ibidem: 128.

${ }^{63}$ Los créditos comerciales financiaban los déficit comerciales, los créditos al desarrollo se utilizaban para financiar proyectos productivos específicos, como los trabajos en el puerto de La Habana, las obras de riego y drenaje, la modernización de la industria azucarera, las 
Tanto Cuba como la URSS si desplazaran sus respectivas exportaciones e importaciones de azúcar al mercado mundial, el precio promedio del mercado mundial — si cambiara - no cambiaría mucho [...] por lo cual es razonable comparar el precio del azúcar en el mercado mundial con el precio preferencial ${ }^{64}$.

También Ritter determina el subsidio al azúcar como diferencia entre el precio pagado por la URSS a Cuba y el precio del mercado mundial, multiplicado por las exportaciones netas ${ }^{65}$ de azúcar y «el valor del subsidio petrolero como diferencia entre el precio del mercado mundial y el precio soviético multiplicado por las exportaciones netas de petróleo de la URSS a Cuba (tomando en cuenta las reexportaciones cubanas de petróleo soviético)» ${ }^{66}$. Obviamente el autor se enfrenta al problema de la tasa de cambio entre peso cubano y dólar estadunidense y considerando la sobrevaluación del peso a la tasa de cambio oficial, sugiere un cambio artificial (dos pesos por un dólar) «probablemente más realista del tipo de cambio oficial desde el punto de vista de la paridad del poder de compra y del libre mercado» y afirma que no pretende decir «que este pueda ser considerado el tipo de cambio adecuado», y que es «muy difícil prever de manera aceptable cuanto sería actualmente este tipo de cambio o cuanto sería si Cuba participara plenamente y abiertamente en la economía internacional sin la relación preferencial con la URSS» ${ }^{67}$.

El trabajo de Ritter es interesante. El autor evidencia algunos problemas (el tipo de cambio, los ingresos para Cuba derivados de la comercialización de azúcar y petróleo ${ }^{68}$ y trata de dar una solución, por eso merece nuestro interés. No obstante, la manera en la cual procede provoca, en mi opinión, el efecto contrario, haciendo aún más visible el problema: está claro que suponer una tasa de cambio de un dólar por dos pesos cubanos solo en base a la consideración que «es probablemente más realista que el tipo de cambio oficial desde el punto de vista de la paridad del poder de compra y del libre mercado ${ }^{69}$ no es un criterio científicamente aceptable. Se puede concluir que Ritter no enfrenta seriamente la «thorny issue» de la verdadera tasa de cambio después de haber

investigaciones geológicas, los proyectos de desarrollo de distintas ramas industriales, del transporte, de la agricultura y de la geología (Rodríguez, 1992a: 66).

${ }^{64}$ Ritter, 1990: 129.

${ }^{65}$ Se restan de las exportaciones de azúcar a la URSS las reexportaciones de azúcar que como hemos visto en el párrafo anterior, Cuba compraba en otros países para mantener las entregas pactadas con los soviéticos, cuando no lograba hacerlo con su producción interna.

${ }_{66}$ Ritter, 1990: 129.

67 Idem.

${ }^{68}$ Por primera vez hay un intento de separar los ingresos por comercialización de las otras formas de subsidio; el error está en la manera en la cual las distintas componentes vienen agregadas.

${ }^{69}$ Ritter, 1990: 129. 
subrayado su importancia, por lo cual, sus tablas de resumen no tienen un respaldo metodológico correcto y no resultan prácticamente útiles, problemas a los cuales el mismo investigador había atribuido la mayor importancia.

En su resumen final, Ritter distingue cinco fuentes de financiamiento: los créditos comerciales, los subsidios implícitos al azúcar y al petróleo, las ganancias por reexportación de azúcar y petróleo. El error metodológico consiste en la necesidad, según el procedimiento del autor, de utilizar el tipo de cambio oficial para agregar las distintas formas de ayuda; en particular, ya subrayamos la manera equivocada y muy distorsionada, para los resultados finales, en la cual Ritter considera las reexportaciones de azúcar y petróleo. A causa de la gran diferencia entre el precio del libre mercado y el precio pagado por los soviéticos, en el periodo 1980-8770, en el cual Ritter hace sus estimaciones, Cuba habría obtenido grandes ganancias, sin embargo, el país se vio forzado a comprar el azúcar en el mercado mundial para, posteriormente, revenderlo a la URSS porque su producción interna no lograba garantizar las entregas pactadas a los soviéticos. También la reexportación de petróleo soviético en el mercado mundial se habría vuelto desfavorable en 1986, mientras sabemos que Cuba hizo lo posible por mantener este importante acceso a la moneda libremente convertible también sucesivamente, por la dificultad que encontraba en generar productos competitivos en los mercados internacionales.

Según Pérez López ${ }^{71}$ en el trabajo de Domínguez ${ }^{72}$ se evita la «thorny issue» del tipo de cambio, porque el autor no realiza la medición en dólares y usa los datos en pesos del AEC. Hay que precisar que el precio oportunidad del azúcar cubano viene determinado por Domínguez sobre la base de los datos AEC, donde los pagos en divisa han sido transformados en pesos a las tasas de cambio oficiales ${ }^{73}$. Bajo otro semblante, se vuelve a manifestar el mismo problema. En un primer momento Pérez López consideró innovador el trabajo de Domínguez, pero terminando el artículo concluye: «la estimación de los subsidios soviéticos a Cuba [...] incluyendo la cuestión de cual sea el tipo de cambio adecuado para pasar de una divisa a otra es claramente un área que necesitaría de una investigación empírica y teórica adicional» ${ }^{74}$.

${ }^{70}$ Por ejemplo, en 1985 en el mercado mundial la tonelada de azúcar se cotizaba en 81,92 pesos cubanos (a la tasa de cambio oficial que en ese año era de un peso por un dólar), mientras los soviéticos la pagaron 986,4 pesos.

${ }^{71}$ Pérez López, 1991: 167.

72 Domínguez, 1978: 291.

${ }^{73}$ Las transacciones con España, Canadá, y Japón, los países tomados en cuenta por Domínguez, se realizaban en moneda convertible (con la excepción de España, en algunos años).

${ }^{74}$ Pérez López, 1988: 145. 


\section{3b. Una evaluación crítica}

Ya tuvimos la oportunidad de evidenciar que el intento de sumar todos los intercambios comerciales y financieros de Cuba con la Unión Soviética, sin tomar en cuenta su impacto diferenciado sobre la economía cubana según fueran en moneda fuerte o ligera, puede llevar a razonamientos incoherentes, sobre todo en aquellas actividades donde se entrelazan ingresos/gastos en moneda convertible y en moneda convenio (reexportaciones de petróleo) o viceversa (reexportaciones de azúcar). Todo eso asume aún mayor relevancia si se quieren dar fundamentos metodológicos al problema de la estimación del subsidio soviético a Cuba y entender cómo y en qué medida impactó sobre la economía cubana.

Los trabajos que hemos considerado en la sección anterior, tratan de medir en dólares el subsidio y se enfrentan al problema de la tasa de cambio ya que, vale la pena recordarlo, los intercambios comerciales y financieros entre los dos países en su gran mayoría eran en moneda convenio (rublos transferibles); al mismo tiempo estos autores concuerdan, cayendo en evidente contradicción, sobre el carácter «tied» de este subsidio que obligaba Cuba a comprar en los países del CAME, los únicos que aceptaban pagos en rublos transferibles.

Medir en dólares el subsidio soviético requiere la construcción de un tipo de cambio peso/dólar económicamente significativo, pero los expertos sobre Cuba que lo han intentado utilizan el tipo de cambio oficial que unánimemente no se considera apropiado ${ }^{75}$. Y lo que es más importante, transformar en dólares el subsidio soviético, significa renunciar a considerar su carácter «tied» que le confiere el hecho de expresarse en rublos transferibles.

Nuestra opinión es que más allá de un debate acerca del tipo de cambio correcto que se pueda utilizar, el subsidio soviético se tiene que cuantificar en términos de los productos que lo componen, o sea los que provenían de la URSS.

En todos estos trabajos que tratan de medir el subsidio soviético a Cuba, se pone el problema del tipo de cambio, porque: 1) los autores quieren llegar a estimaciones en dólares y, como hemos visto, los intercambios se financiaban mayormente en rublos transferibles; 2) el subsidio al azúcar se calcula como diferencia entre el precio pagado por los soviéticos y el precio del mercado libre (o el precio

75 Por ejemplo, Mesa Lago (2000: 256) afirma que hacer «comparaciones históricas del comercio exterior de Cuba es complicado entre 1971-1985 porque el peso cesó de ser cotizado a la par con el dólar norteamericano, ya que el gobierno cubano lo fijó de manera arbitraria por encima a 0,71 pesos por un dólar en $1980 \ldots$ este tipo de cambio era artificial». Mi opinión es que este tipo de cambio siempre fue artificial y arbitrariamente fijado, por tanto, no es utilizable para determinar los beneficios que Cuba derivaba en el comercio con los países del CAME, especialmente con la Unión Soviética. 
preferencial de Estados Unidos) y el subsidio petrolero como diferencia entre el precio pagado en el mercado mundial y el precio pagado por Cuba a la URSS.

Dado que la URSS pagaba casi todo el azúcar cubano en rublos transferibles y con ellos Cuba le compraba el petróleo y otros productos, es necesario transformar los rublos en dólares para poder comparar el precio de los dos productos en el mercado mundial. Además una parte del subsidio soviético era constituido por créditos comerciales y al desarrollo, por tanto, se expresaba en rublos transferibles, y se tenía que transformar en dólares. Por eso se presentaba necesariamente la «thorny issue» de la tasa de cambio.

Nuestra hipótesis es que todas estas mediciones son innecesarias para cuantificar la importancia del subsidio soviético a Cuba y, sobre todo, conceptualmente no son correctas, porque para llegar a una medición en dólares, dejan de lado el carácter «tied» del subsidio.

En conclusión, el subsidio soviético era «tied», o sea, obligaba el gobierno cubano a comprar en el área del CAME los productos que se incluían en los acuerdos comerciales, que no necesariamente eran los más deseados. Por tanto: 1) ninguna medición en dólares puede ser útil para cuantificar el subsidio y el indicador más correcto, en el plano teórico, porque evita el problema del tipo de cambio, es la relación entre subsidio e importaciones de la URSS, que es un numero puro ya que ambos agregados se expresan en rublos transferibles; 2) en el caso de las reexportaciones de petróleo se cruzaban flujos en moneda convenio y moneda convertible, que se deben analizar por separado (lo que hicimos en la sección anterior).

\section{3c. La propuesta}

El método que voy a exponer pretende dar solución a los tres problemas señalados por Zimbalist y Brundenius en las estimaciones de la CIA.

La ausencia de un tipo de cambio que tenga significado económico y los problemas para derivarlo y, aún más importante, la irracionalidad de expresar en dólares la ayuda soviética, me indujeron a buscar un camino menos directo, para determinar el nivel de esa ayuda y sus repercusiones sobre la economía cubana.

Retomando la sugerencia de Turits ${ }^{76}$, según el cual «cada estimación confiable de los subsidios soviéticos tendrá que examinar cuidadosamente el complejo nexo de las transacciones en moneda débil y fuerte», proponemos dividir, en las relaciones cubano-soviéticas, la parte del intercambio en moneda fuerte de la otra en moneda convenio, o sea, en rublos transferibles. Las

\footnotetext{
${ }^{76}$ Turits, 1987: 174.
} 
observaciones de Turits sobre la importancia de profundizar las relaciones URSS-Cuba y el bajo poder adquisitivo en el mercado mundial de las exportaciones cubanas, en relación a las importaciones de la URSS, «si se valoraran a los precios del mercado mundial [...] Cuba no hubiese comprado ni siquiera el petróleo con los ingresos del azúcar» ${ }^{77}$, conjuntamente a la sencilla composición de las exportaciones cubanas y el hecho de que productos como el níquel, el azúcar y el petróleo, cotizables en el mercado mundial, constituyen más de $2 / 3$ del intercambio, nos sugirieron el siguiente procedimiento ${ }^{78}$ :

a) Multiplicar las exportaciones físicas de azúcar y níquel a la URSS por el precio de estos productos en el mercado mundial, determinando su valor en dólares. Restando de las exportaciones cubanas a la URSS, por las razones explicadas en la sección 2, las reexportaciones de petróleo, el azúcar y el níquel representaron en promedio en el periodo 1976-88, el 97,5\% de las exportaciones a la URSS.

b) Determinar el poder de compra de los dos productos en el mercado mundial, en términos de petróleo, o sea la cantidad de petróleo que Cuba hubiese podido comprar en el mercado mundial si hubiera vendido allí el azúcar y el níquel.

c) Multiplicar esas cantidades de petróleo por su precio c.i.f. (coste, seguro y flete $)^{79}$ en pesos no convertibles, en el intercambio CubaURSS, determinando así el valor, en base al precio preferencial pagado por los cubanos, del petróleo que Cuba hubiese podido comprar exportando el azúcar y el níquel en el mercado mundial.

d) Restar de las importaciones cubanas, medidas en pesos, tanto el valor de las otras exportaciones cubanas a la URSS - en promedio solo el $2,5 \%$ de las totales ${ }^{80}$ - como el valor del petróleo calculado en el inciso anterior, que constituyen la parte de las importaciones soviéticas que Cuba hubiese efectivamente comprado. De esta manera se

${ }^{77}$ Idem.

${ }^{78}$ En el anexo presentamos el método de medición del subsidio con un ejemplo numérico sencillo.

79 Usar el precio c.i.f. (incluye los costos de seguro y flete) para el petróleo soviético es la única posibilidad porque los datos AEC de las importaciones incluyen los gastos en seguros y de transporte que eran muy relevantes como observan varios cubanologos (véase por ejemplo Recarte 1981: 150 o Gey, 1987: 80) y en su mayoría a cargo de la URSS. El uso del precio c.i.f. para el petróleo soviético, causaría entonces una distorsión hacia abajo de la estimación del subsidio.

${ }^{80}$ Hubiera sido preferible valorar también las otras exportaciones a la URSS al precio del mercado mundial, como para el níquel y el azúcar, pero se trata de productos difíciles de valorar a esos precios. Considerando que su valor es muy reducido, esta operación no influencia sensiblemente los resultados finales. 
determina la parte no pagada de las importaciones, y una primera medición de la ayuda soviética a Cuba que llamaré subsidio.

e) A partir de la información disponible determinar los valores anuales de créditos al desarrollo, considerando uniforme su distribución en el tiempo en los años cuando solo hay una información agregada (por ejemplo, entre 1975-85 solo hay un dato total, para todo el periodo). Añadirles los créditos comerciales, determinados sumando los déficits anuales que Cuba siempre sostuvo ${ }^{81}$.

f) El último paso consiste en subdividir el subsidio global en sus dos componentes fundamentales: i) la parte explicita del subsidio vía créditos; ii) la parte implícita vía precios preferenciales, calculados como diferencia entre el subsidio total y el primero. Como veremos eso es importante para un análisis crítico de los intentos de estimación de la literatura, que a veces tratan de llegar a conclusiones sobre la evolución del subsidio soviético a partir de un análisis parcial de una de sus componentes, la ayuda implícita en los precios preferenciales o una sola parte de ella, la que se mide sobre el precio preferencial pagado por el azúcar. Veremos que a veces a las oscilaciones improvisas de uno de los componentes del subsidio, siguen las variaciones en el sentido opuesto de la otra, con un efecto equilibrador sobre el dato global. Evitamos pues, sacar conclusiones sobre la evolución de la ayuda económica a Cuba sobre la base de un examen parcial de una sola parte de ella.

El subsidio soviético a Cuba se determina así como suma de distintos componentes, todos expresados en su propia unidad de medición (pesos no convertibles). Sin tener que utilizar el tipo de cambio, es posible, por ejemplo, dividir este subsidio entre el total de las importaciones de la URSS y también sobre el ingreso nacional creado (INC), el indicador más cercano al producto interior bruto (PIB) en la contabilidad que se adoptaba en los países socialistas. De esta manera se da un fundamento metodológico al intento de estimación de Ritter ${ }^{82}$, sin chocar, como en su caso, con la «thorny issue» del tipo de cambio. En la tabla 5 resumimos los resultados obtenidos.

${ }^{81}$ En relación a los créditos comerciales precisamos: a) no se incluyen entre las exportaciones a la URSS las reexportaciones de petróleo, por lo que se aclaró en la sección 2; b) se supone que no hubo ningún reembolso por parte cubana, dada la ausencia de información oficial al respecto (en un intercambio entre el autor y Pérez López, este confirma que estos reembolsos pueden haber sido mínimos y «se pueden ignorar a efecto de estudios globales»); c) no consideramos los intereses que se acumulaban (en los créditos comerciales la tasa de interés era inferior al $4 \%$ anual, y en los créditos al desarrollo era del 2 \%) (Banco Nacional de Cuba, 1982: 15).

${ }^{82}$ Ritter, 1990: 132. 
TABla 5: Subsidio SOVIÉTICO A CUBA EN RELACiÓN AL TOTAL DE LAS IMPORTACIONES (SUBSIDIO 1) Y AL INC ${ }^{83}$ CUBANO (SUBSIDIO 2)

\begin{tabular}{|c|c|c|c|c|c|c|}
\hline \multicolumn{3}{|c|}{ Subsidio 1 (\% de las importaciones) } & \multicolumn{3}{c|}{ Subsidio 2 (\% del INC) } \\
\hline Años & Total (\%) & Precios (\%) & Préstamos (\%) & Total (\%) & Precios (\%) & Préstamos (\%) \\
\hline 1968 & 74,8 & 18 & 56,8 & 11,5 & 2,8 & 8,7 \\
\hline 1969 & 67,7 & 2,5 & 65,2 & 10,8 & 0,4 & 10,4 \\
\hline 1970 & 37,9 & 14,5 & 23,4 & 6,2 & 2,4 & 3,8 \\
\hline 1971 & 66 & 7,6 & 58,4 & 10 & 1,1 & 8,9 \\
\hline 1972 & 114,8 & $-8,3$ & 123,1 & 16,3 & $-1,2$ & 17,5 \\
\hline 1973 & 43,3 & 2,1 & 41,2 & 5,2 & 0,3 & 4,9 \\
\hline 1974 & 56,6 & 35,7 & 20,9 & 7,8 & 4,9 & 2,9 \\
\hline 1975 & 35,2 & 68,1 & $-32,9$ & 5,4 & 10,4 & -5 \\
\hline 1976 & 90,8 & 79,9 & 10,9 & 15,7 & 13,8 & 1,9 \\
\hline 1977 & 87,7 & 82,2 & 5,5 & 17,5 & 16,4 & 1,1 \\
\hline 1978 & 88,5 & 82,4 & 6,1 & 20,6 & 19,2 & 1,4 \\
\hline 1979 & 98,3 & 80,5 & 17,8 & 24,6 & 20,1 & 4,5 \\
\hline 1980 & 89,3 & 52,9 & 36.4 & 27,2 & 16,1 & 11,1 \\
\hline 1981 & 91,4 & 50 & 41,4 & 25,7 & 14 & 11,7 \\
\hline 1982 & 93,4 & 66 & 27,4 & 28,9 & 20,4 & 8,5 \\
\hline 1983 & 95,2 & 65,4 & 27,6 & 31 & 21,8 & 9,2 \\
\hline 1984 & 96,4 & 61,2 & 34 & 33,2 & 21,4 & 11,8 \\
\hline 1985 & 86,4 & 63,6 & 32,8 & 36,6 & 24,2 & 12,4 \\
\hline 1986 & 89,1 & 45,1 & 41,3 & 33,1 & 17,3 & 15,8 \\
\hline 1987 & 69,3 & 44,7 & 44,4 & 36,6 & 18,3 & 18,3 \\
\hline 1988 & 69,3 & 24,2 & 45,1 & 27,4 & 9,6 & 17,8 \\
\hline & & & Promedios $(\%)$ & & \\
\hline $1968-75$ & 62 & 17,5 & 44,5 & 9,15 & 2,64 & 6,51 \\
\hline $1976-88$ & 89,9 & 61,4 & 28,5 & 27,55 & 17,89 & 9,65 \\
\hline $1968-88$ & 79,3 & 44,7 & 34,6 & 20,54 & 12,08 & 8,46 \\
\hline & & 6 & & & & \\
\hline
\end{tabular}

Fuente: Rodríguez (1992a: 63) para el intercambio Cuba-URSS; Banco Nacional de Cuba (1986: 23; 1987: 23; 1988: 15; 1989: 15) para las reexportaciones de petróleo; Anuario Estadístico de Cuba (1972: 214-216; 1977: 172, 179; 1981: 207-208 y 211-212; 1985: 432, 433, 440, 441; 1988: 467, 485) para las exportaciones de níquel y azúcar en cantidad y valor; Anuario Estadístico de Cuba (1972: 222-223; 1977: 188-189; 1981: 217-218; 1985: 452-453; 1988: 486-487) para las importaciones de petróleo. Las exportaciones en 1988 de níquel a la URSS, así como las importaciones de petróleo, se han considerado constantes con respecto a 1987, en base a la información de Rodríguez (1992b: 9). International Financial Statistics Yearbook (1993: 166-167) para los precios en el mercado mundial de azúcar, níquel y petróleo. Rodríguez (1992a: 66) para los créditos al desarrollo.

${ }^{83}$ En el sistema de contabilidad socialista, utilizado en Cuba, el ingreso nacional creado (INC) era el indicador más cercano al producto interno bruto (PIB) en uso en los países capitalistas. 
La estimación del subsidio soviético a Cuba se aplicó al periodo 1968-88, para el cual existe la necesaria información. El primer anuario estadístico fue publicado en Cuba en 1972, con datos a partir de 1968.

Construimos dos indicadores: el subsidio relacionado al total de las importaciones de la URSS (subsidio 1) y el otro que se divide entre el INC cubano (subsidio 2). El primero nos permite aclarar la transferencia física de bienes que tuvo lugar, porque en el AEC se encuentran desglosados producto por producto $^{84}$ alrededor del $80 \%$ del total de importaciones, mientras que el segundo nos hace entender el impacto que tuvo sobre la economía cubana. El subsidio total se ha dividido en sus dos componentes: la parte explicita (directamente observable sumando los créditos comerciales y al desarrollo) y la parte implícita (a través de los precios preferenciales de las principales exportaciones e importaciones), cuya determinación necesitó la construcción del procedimiento que acabamos de describir.

Se puede dividir el periodo en dos, con el año 1975 como parteaguas, que se diferencian no solo por el valor total del subsidio, muy superior en el segundo periodo, sino también por un cambio radical en su composición. El subsidio por préstamos prevalecía entre 1968-1975, siendo el 44,5\% del total de las importaciones mientras el subsidio implícito era del 17,5\%, sin embargo, éste crece en el segundo periodo hasta el 61,4\% de las importaciones, con el primero que baja hasta el 28,5\%. Esto significa que entre 1976-88 el mecanismo de precios preferenciales fue la primera vía de financiamiento de la economía cubana por parte de la URSS, por lo cual hay que hacer hincapié en la importancia para Cuba del acuerdo de 1975, cuando obtuvo precios mucho más favorables para la venta de azúcar y la compra de petróleo. Precisamente con el nuevo acuerdo de 1975 se abre una nueva fase en las relaciones comerciales entre los dos países, aún más favorable para Cuba que el periodo anterior.

Si hacemos referencia al impacto del subsidio sobre el INC de Cuba, se confirma que a lo largo de todo el periodo la parte implícita del subsidio es la que prevalece, siempre notando un cambio radical en 1975. Antes de 1975 el subsidio total era el 9,2\% del INC y se multiplica por tres entre 1976-88, llegando al 27,5\%. Eso se explica sobre todo por el aumento del subsidio implícito que en el primer periodo era un cuarto del total y llega a dos tercios en el segundo.

Domínguez ${ }^{85}$ se sorprendía de que F. Castro no se quejara del empeoramiento de los términos de intercambio a principio de los años ochenta, sin

${ }^{84}$ Entre las principales importaciones estaban el petróleo, los fertilizantes, el trigo, madera, plantas completas, tractores, carros y autopartes, láminas de acero, neumáticos etc.

${ }^{85}$ Domínguez, 1978: 88. 
embargo el líder cubano era probablemente consciente de que en su conjunto las relaciones económicas entre los dos países nunca habían sido tan favorables para $\mathrm{Cuba}^{86}$. Por eso destacar las pérdidas ${ }^{87}$ para Cuba en las relaciones económicas con la URSS en los años ochenta es totalmente engañoso: la muy generosa ayuda soviética se mantuvo hasta 1987 y considerada en sus múltiples formas logró los niveles más altos precisamente en ese periodo. Tampoco la hipótesis de Ritter ${ }^{88}$ según la cual las relaciones con los soviéticos empeoran para Cuba es válida, porque hasta 1987 se mantienen los altos valores alcanzados en el primer quinquenio de los ochenta.

Esta observación asume especial importancia porque futuros miembros del gobierno cubano ${ }^{89}$ y académicos extranjeros especializados en Cuba como Ritter, atribuyen a la reducción de la ayuda soviética la crisis que empezó en 1986 y dio lugar al proceso de rectificación. De acuerdo con Mesa Lago ${ }^{90}$, la causa principal de la nueva ofensiva fue la voluntad política de F. Castro de acabar con el poder de los tecnócratas y su intento de planificar la economía con el SDPE, además de un empeoramiento del contexto internacional (la reducción del precio del petróleo en 1986 perjudicó a Cuba que, como vimos, reexportaba parte del petróleo soviético, así como la devaluación del dólar hacia otras divisas en las cuales Cuba había contraído relevantes préstamos).

\section{CONCLuSiones}

La economía cubana ha sido fuertemente subsidiada por la Unión Soviética, en el periodo revolucionario hasta que empezaron los cambios políticos que determinaron el derrumbe del campo socialista (1989) y la desaparición de la URSS (1991). Sin embargo, hemos aclarado que ningún estudioso de la economía cubana ha logrado dar fundamentos metodológicos claros y aceptables para medir este subsidio.

${ }^{86}$ A mitad de los ochenta, en el intento de desacreditar el Sistema de Dirección y Planificación de la Economía (SDPE), F. Castro (citado en Gey, 1987: 80) subrayó: «disponemos de abundantes recursos, ya que lo que recibimos solo de la URSS en un quinquenio incluyendo mercancías, transporte y créditos, es más de 20 mil millones de rublos».

${ }^{87}$ Algunos análisis en este sentido son los de Rodríguez (1992b: 4) y Zimbalist y Brundenius (1989: 152) que subrayan el empeoramiento de los términos de intercambios para Cuba en el comercio con la URSS sobre todo después de 1980, en el claro intento de minimizar los beneficios para la economía cubana.

${ }_{88}^{8}$ Ritter, 1990: 133.

89 Rodríguez, $1992 \mathrm{a}: 57$.

90 Mesa Lago, 1992: 29. 
En este trabajo hemos construido un nuevo método, que nos parece efectivo y relativamente sencillo, para dar una estimación del subsidio y de su impacto sobre la economía cubana, que pretende superar las limitaciones anteriores. Hemos empezado cuantificando la importancia para Cuba de las reexportaciones de azúcar, luego aclaramos el papel que las reexportaciones de petróleo jugaban para garantizar los ingresos en moneda convertible tan imprescindibles para el gobierno cubano en la década de los ochenta. Demostramos, además, que en la contabilidad de Cuba, las reexportaciones de petróleo se registraban como exportaciones a la URSS, probablemente para maquillar los déficits comerciales con ese país. Se trata obviamente de una operación contable inaceptable, pues Cuba importaba el petróleo de la URSS, y lo que no consumía lo reexportaba a los países europeos que lo pagaban en moneda convertible.

Aplicando nuestro método pudimos calcular el subsidio en términos de los productos soviéticos importados, lo cual permite tener una idea bastante precisa y concreta de su nivel e importancia para la economía cubana. Además, logramos dividir y medir el subsidio en sus dos componentes, el llamado subsidio explicito (los créditos comerciales y al desarrollo) y el subsidio implícito, que se generaba a través de los precios preferenciales, sobre todo de las exportaciones cubanas de azúcar y níquel y las importaciones de petróleo.

Los resultados muestran la gran relevancia que el subsidio soviético siempre tuvo para la economía cubana en el periodo 1968-88, y su tendencia creciente. En relación al total de las importaciones de la URSS, el subsidio fue del $62 \%$, aumentando hasta casi el $90 \%$ entre 1976-88. En promedio el 79,3 \% de esas importaciones no venían cobradas por los soviéticos, tomando como referencia lo que Cuba hubiera importado en el mercado mundial. En otras palabras, si Cuba no hubiese gozado de un trato preferencial en el comercio con los soviéticos y hubiese comprado en el mercado mundial los productos que necesitaba, hubiese podido comprar solo el $20 \%$ de lo que efectivamente importó de la URSS. También se observa un cambio en la composición de dicho subsidio, que en el primer periodo estaba compuesto por créditos comerciales en prevalencia, sin embargo en el segundo periodo $2 / 3$ del total transitaba a través del mecanismo de los precios preferenciales. En relación al INC cubano, el subsidio se triplicó entre el primer periodo y el segundo, y en promedio es del 20,5 \%. El subsidio a través de precios, minoritario en el primer periodo, es el que más crece, llegando al 17,9 \% del INC entre 1976-88. Se confirma la intuición 
de Ritter ${ }^{91}$, que colocaba el subsidio soviético entre el 10 y $40 \%$ del INC cubano. El valor que nosotros estimamos con precisión y una clara metodología, es similar a la contracción del INC reconocida sucesivamente por las autoridades cubanas entre el cese del apoyo soviético y la fase más aguda del periodo especial (1994).

\section{ANEXo}

Los siguientes datos simulan el intercambio entre Cuba y la URSS:

a) Exportaciones cubanas de azúcar + níquel en el mercado mundial $=100$ dólares estadounidenses.

b) En el mercado mundial con 100 dólares estadounidenses se compran 10 toneladas de petróleo.

c) En el intercambio Cuba-URSS 10 toneladas de petróleo cuestan 120 pesos cubanos (PCU).

d) Otras exportaciones cubanas a la URSS $=5$ PCU.

e) Créditos al desarrollo $=20$ PCU.

f) Exportaciones cubanas $=200 \mathrm{PCU}$.

g) Importaciones cubanas $=300$ PCU.

h) Subsidio total $=\mathrm{g}-\mathrm{c}-\mathrm{d}=(300-120-5)$ PCU $=175$ PCU.

Subsidio explicito (préstamos) $=\mathrm{g}-\mathrm{f}+\mathrm{e}=(300-200+20) \mathrm{PCU}=120 \mathrm{PCU}$.

Subsidio implícito (precios preferenciales) $=$ Subsidio total - subsidio explicito $=(175-120)$ PCU $=55$ PCU.

Subsidio total $/$ Importaciones $=175 / 300=58,3 \%$.

\section{BiBLIOGRAFía}

Anuario Estadístico de Cuba, La Habana, Comité Estatal de Estadística, 1972, 1977, 1981, 1985, 1988, 1989.

Banco Nacional de Cuba, Informe Económico, La Habana, 1982, 1985, 1986, 1987, 1988, 1989, 1990.

Castro, Fidel, La revolución de octubre y la revolución cubana. Discursos 1959-1977, La Habana, edición del departamento de orientación revolucionaria del Comité Central del PCC, 1977.

\footnotetext{
${ }^{91}$ Ritter, 1990: 132.
} 
CIA, The cuban economy: a statistical review, 1968-76, Washington, December 1976.

Dominguez Jorge, Cuba: order and revolution, Cambridge, Harvard University Press, 1978.

Dominguez Jorge, To make a world safe for revolution, Cambridge, Harvard University Press, 1989.

Fernández Font, Marcelo, Cuba y la economía azucarera mundial, La Habana, Editorial Pueblo y Educación, 1989.

García Fernández, Francisco y Sovilla, Bruno, "Características y vulnerabilidades del sector exportador cubano en el periodo revolucionario (1959-2013)", América Latina en la Historia Económica, 24/1, (Ciudad de México, 2017): 140-170.

Gey Peter, "The Cuban economy under the system of management and planning: success or failure?", P. Gey, J. Kosta y W. Kaiser (eds.), Crisis and reform in socialist economies, Boulder, Westview Press, 1987: 71-98.

International Financial Statistics Yearbook, Washington, Fondo Monetario Internacional, 1993: 166-167.

Marrese, Michael y Vanous, Jan,, Soviet subsidization of trade with Eastern Europe, Berkeley, Univiersity of California, Institute of International Studies, 1983.

Mesa Lago, Carmelo, "Problemas estructurales, política económica y desarrollo en Cuba 1959-70", Desarrollo Económico, 13/51 (Buenos Aires, 1973): 533-583.

Mesa Lago, Carmelo, "Causas, magnitud y alternativas de la crisis económica de Cuba en los 90", Cuadernos del Este, 6 (Madrid, 1992): 25-38.

Mesa Lago, Carmelo, Market, Socialist and Mixed Economies: Comparative Policy and Performance--Chile, Cuba and Costa Rica, Baltimore, John Hopkins University Press, 2000.

Mesa Lago, Carmelo y Gil, Fernando, "Vingt and de relations economiques entre 1' URSS et Cuba”, Le courier des Pays de l'Est, 355 (París, diciembre 1988): 52-74.

Pérez López, Jorge, "Sugar and petroleum in Cuban-Soviet terms of trade", Mesa Lago y Blasier ed. Cuba in the world, Pittsburgh, University of Pittsburgh Press, 1979.

Pérez López, Jorge, "Cuba as an oil trader", Caribbean Review, 15 (Trinidad y Tobago, 1986): 26-44.

Pérez López, Jorge, "Cuban-Soviet sugar trade: price and subsidy issues", Bulletin of Latin American Research, VII/1 (Reino Unido, 1988): 123-147.

Pérez López, Jorge, The economics of cuban sugar, Pittsburgh, University of Pittsburgh Press, 1991.

Pérez López, Jorge, "The Cuban economy: rectification in a changing world", Cambridge Journal of Economics, 16 (Cambridge, 1992): 113-126. 
Radell, Willard W. Jr, "Cuban soviet sugar trade, 1960-76: how great was the subsidy?", The Journal of Developing Areas, 17 (Baltimore, 1983): 365-382.

Recarte, Alberto, Cuba: economía y poder (1959-1980), Madrid, Alianza editorial, 1980.

Ritter, Archibald R. M., "Cuba's convertible currency debt problem”, Cepal Review, 36 (Santiago de Chile, 1988): 115-140.

Ritter, Archibald R. M., "The cuban economy in the 1990s: external challenges and policy imperatives", Journal of interamerican studies and world affairs, XXIII/3 (Miami, 1990): 117-148.

Rodríguez, José Luis, "Un enfoque burgués del sector externo de la economía cubana”, Cuba Socialista, 14 (La Habana, 1985): 78-103.

Rodríguez, José Luis, "Las relaciones económicas Cuba-URSS, 1960-1985", Temas de la economía mundial, 17 (La Habana, 1986).

Rodríguez, José Luis, Estrategia del desarrollo económico en Cuba, La Habana, Ed. Ciencias sociales, 1990.

Rodríguez, José Luis, "Las relaciones económicas entre Cuba y la antigua URSS: evaluación y perspectivas", Cuadernos del Este, 5 (Madrid, 1992a): 51-66.

Rodríguez, José Luis, "Las relaciones económicas entre Cuba y la antigua URSS: 1959-1990", Boletín del CIEM, 7 (La Habana, 1992b): 2-12.

Rodríguez, José Luis, "La deuda externa cubana: una evaluación actual (I)", Boletín del CIEM, 10 (La Habana,1993): 2-11.

Sovilla, Bruno y García Fernández, Francisco, "La economía cubana: entre voluntarismo e intentos de planificación (1959-2012)", Revista mexicana de Ciencias Políticas y Sociales, LVIII/219 (México, 2013): 157-188.

Torres, Olga Ester R., "El desarrollo de la economía cubana a partir de 1959", Comercio Exterior de México, XXXI/3 (México, 1981): 284-295.

Turits, Richard, "Trade, debt and the cuban economy", World development, XV/1 (Estados Unidos, 1987): 163-180.

Zimbalist, Andrew y Brundenius, Claes, The Cuban economy. Measurement and analysis of a socialist performance", Baltimore / London, The Johns Hopkins University Press, 1989.

Fecha de recepción: 4 de noviembre de 2019.

Fecha de aceptación: 5 de marzo de 2020. 


\section{How can Soviet aid to the Cuban economy be measured? A new proposal}

Trade between the former Soviet Union (USSR) and Cuba during the period before the collapse of the Socialist bloc has been the subject of many studies, which have attempted to quantify that commerce in US dollars without taking into account the peculiarities of the relations between the Socialist countries that were members of the Council for Mutual Economic Assistance (CMEA). This research has drawn on official sources (from the Office of Statistics and the Banco Nacional de Cuba) to remedy the methodological errors that result from overlooking those peculiarities, and proposes a new metric for the aid received by Cuba from the USSR.

KEY WORDS: subsidy; exchange rate; sugar; oil; re-exports. 